\title{
Réforme des structures de la FMH: c'est parti!
}

\author{
Jacques de Haller, Président de la FMH
}

Madame, Monsieur, cher-es Collègues, Le projet d'une réforme des structures de la FMH a fait l'objet de plusieurs décisions de la Chambre médicale, l'an dernier (la Chambre médicale est le législatif de la FMH); il est maintenant sur les rails.

Ce projet est réellement important; il y a en effet en arrière-plan de ce processus de réforme à la fois notre capacité à agir dans les années qui viennent, mais aussi toute la question de la cohésion et de la représentativité de la FMH: il faut que dans le futur, chacun des groupes qui constituent notre organisation s'y sente pris en compte et correctement représenté.

Un Comité de pilotage a été constitué, soigneusement représentatif de tous les groupes de spécialités, y compris l'ASMAC, de toutes les langues, etc.; ce Comité a commencé son travail en décembre dernier et compte l'achever à la fin de l'été - il s'agit donc d'un projet qui va très rapidement se concrétiser, même s'il entraînera certainement des changements profonds dans notre organisation.

Parallèlement à cette réforme des structures se fait une réflexion du Comité central sur la stratégie de la FMH, pour les 5-10 ans à venir; les résultats de cette réflexion du Comité central influenceront évidemment le travail du Comité de pilotage, à qui ils seront communiqués, et ils seront aussi soumis en temps utiles aux organes décisionnels de la FMH.

Le Comité de pilotage de la réforme des structures, lui, a prévu de commencer par évaluer la situation actuelle et les besoins, au niveau structurel, puis de continuer en formulant des propositions qui seront mises en consultation, et de terminer à l'été prochain par des recommandations concrètes, notamment statutaires.
L'évaluation de la situation actuelle et des besoins va se faire sur la base d'un questionnaire et c'est justement le but de cet article que de l'annoncer:

Toutes les personnes détenant un mandat au sein de la FMH vont recevoir ces prochains jours un document à renvoyer avant fin février, visant à connaître leurs opinions, leurs idées, et à préciser les résultats à obtenir par la réforme des structures.

Les destinataires prévus de ce questionnaire sont plusieurs centaines; il s'agit:

- des délégué-es à la Chambre médicale;

- des membres de la CFPC;

- des membres de la Commission financière et du Bureau du Conseil suisse de déontologie;

- des membres du Comité central;

- des Comités des sociétés cantonales de médecine et des Comités des sociétés de discipline médicale;

- des membres du Comité de l'ASMAC, de l'ASMPH et de toutes les diverses organisations «autorisées à intervenir à la Chambre médicale».

Les résultats de ce sondage seront dépouillés par les soins de «B'VM», le consultant qui accompagne notre réflexion sur les structures; ils permettront de savoir dans quelle direction et avec quelle ampleur des réformes sont nécessaires, et de faire ensuite les propositions adéquates.

Le Comité central de la FMH et le Comité de pilotage du projet de réforme des structures remercient donc chaleureusement les destinataires de ce questionnaire de l'heure (environ) qu'ils/elles y consacreront: rénover la FMH est un projet fascinant qui, au-delà des difficultés que nous avons vécues ces dernières années, a maintenant besoin de la participation active de chacune et chacun! 